Faculty of Mathematical Sciences

\section{University of Twente}

University for Technical and Social Sciences
P.O. Box 217

7500 AE Enschede

The Netherlands

Phone: +31-53-4893400

Fax: +31-53-4893114

Email: memo@math.utwente.nl

Memorandum No. 1582

Nehari problems and equalizing vectors

for infinite-dimensional systems

O.V. Iftime And H.J. ZwarT

APRIL 2001

ISSN 0169-2690 


\title{
Nehari problems and equalizing vectors for Infinite-Dimensional Systems*
}

\author{
O. Iftime and H. Zwart \\ Faculty of Mathematical Sciences \\ University of Twente \\ P.O. Box 217, 7500AE, Enschede \\ The Netherlands \\ e-mail:\{o.v.iftime, h.j.zwart\}@math.utwente.nl
}

\begin{abstract}
For a class of infinite-dimensional systems we obtain a simple frequency domain solution for the suboptimal Nehari extension problem. The approach is via $J$-spectral factorization, and it uses the concept of equalizing vectors. Moreover, the connection between the equalizing vectors and the Nehari extension problem is given.

Keywords: infinite-dimensional systems, Nehari problem, equalizing vectors, $J$-spectral factorization
\end{abstract}

Mathematics Subject Classification: 93B36, 93C25, 47A68, 47B35.

${ }^{*}$ This paper has been submitted to Systems and Control Letters 


\section{Introduction}

The Nehari problem is naturally formulated in frequency domain: given a matrix-valued function $G$, find the distance from $G$ to the stable matrix-valued functions. The problem of finding $K$ that achieves the minimum distance is called the Nehari extension problem. In this paper we consider the Nehari extension problem together with a special version of this problem, known as the suboptimal Nehari extension problem. This is: given a matrix-valued function $G$ and a $\sigma>0$, find (if it exists) a stable $K$ such that

$$
\|G+K\|_{\infty}=\operatorname{ess} \sup _{\omega \in}\|G(j \omega)+K(j \omega)\|<\sigma .
$$

These problems have received wide attention in the mathematical-, and the systems and control literature (see [1], [3], [6], [7], [9], [11], [16] , [17], [18], [19]). Some control problems can be reduced to a Nehari problem (see e.g. [8], chapter 9). In [5], the suboptimal Nehari extension problem is used, in an essential way, for solving the standard $H_{\infty}$-suboptimal control problem for a class of infinite-dimensional systems. For the solution of the Nehari extension problem, the authors of [5] refer to the abstract results obtained in [2], [3].

Our class of infinite-dimensional systems consists of systems whose impulse responses can be composed in a delta distribution at zero plus an integrable function. For this class of systems we give a direct frequency domain solution for the suboptimal Nehari extension problem. Using similar techniques one can show that the same result holds for the systems considered in [5], i.e., systems whose impulse response is a delta function plus a weighted integrable function. The approach is via $J$-spectral factorization, and uses a recent result obtained in [14]. Via a simple proof, we show that the suboptimal Nehari extension problem is solvable if and only if a certain $J$-spectral factorization exists. The connection between the equalizing vectors and the Nehari extension problem is provided in Section 4.

\section{Preliminaries}

We introduce our class of stable transfer functions via their impulse responses. We say that $f \in \mathcal{A}$ if $f$ has the representation

$$
f(t)= \begin{cases}f_{a}(t)+f_{0} \delta(t), & t \geq 0 \\ 0, & t<0\end{cases}
$$

where $f_{0} \in \mathbb{C}, \int_{0}^{\infty}\left|f_{a}(t)\right| d t<\infty$ and $\delta$ represents the delta distribution at zero. Let $\hat{f}$ denote the Laplace transform of $f$. Then $\hat{\mathcal{A}}$ defined as $\hat{\mathcal{A}}:=\{\hat{f} \mid f \in \mathcal{A}\}$ is our class of stable transfer functions. By the definition of $\mathcal{A}$ it is easy to see that for every $f \in \mathcal{A}, \hat{f}$ is well-defined on $\overline{\mathbb{C}}_{+}:=\{s \in \mathbb{C} \mid \operatorname{Re}(s) \geq 0\}$, it is holomorphic and bounded on $\mathbb{C}_{+}:=\{s \in \mathbb{C} \mid \operatorname{Re}(s)>0\}$, and continuous on $j \mathbb{R}:=\{s \in \mathbb{C} \mid \operatorname{Re}(s)=0\}$. Furthermore, $\hat{\mathcal{A}}$ is a commutative Banach algebra with identity under pointwise addition and multiplication (see [8], Corollary A.7.48).

For (matrix-valued) functions we define $F^{\sim}(s)=[F(-\bar{s})]^{*}$, where ${ }^{*}$ denotes the transpose complex conjugate. We also consider the Wiener algebra

$$
\hat{\mathcal{W}}=\left\{\hat{f} \in L_{\infty} \mid \hat{f}=\hat{f}_{1}+\hat{f}_{2}, \text { with } \hat{f}_{1}, \hat{f}_{2}^{\sim} \in \hat{\mathcal{A}}\right\},
$$

where $L_{\infty}$ is the space of essentially bounded functions, on the imaginary axis. $\hat{\mathcal{W}}$ is a Banach algebra under pointwise addition, multiplication, and scalar multiplication. The elements of 
$\hat{\mathcal{W}}$ are bounded and continuous on the imaginary axis, and their limit at infinity exists. For more properties of $\hat{\mathcal{W}}$ we refer to [4].

The space $H_{2}$ denotes the standard Hardy space on the right-half plane. The space $H_{2}^{\perp}$ is the orthogonal complement of $H_{2}$ with respect to the inner product in the space $L_{2}$ of square integrable functions on the imaginary axis. We denote by $L_{\infty}^{n \times m}, \hat{\mathcal{A}}^{n \times m}, \hat{\mathcal{W}}^{n \times m}$, the classes of $n \times m$ matrices with entries in $L_{\infty}, \hat{\mathcal{A}}, \hat{\mathcal{W}}$, respectively. We omit the size of the matrix when there is no danger of confusion. A square matrix-valued function $G \in \hat{\mathcal{W}}$ is invertible over $\hat{\mathcal{W}}$ if and only if $\operatorname{det} G(j \omega) \neq 0$ for $\omega \in \mathbb{R} \cup\{\infty\}$ (see [4]). We say that a matrix-valued function is bistable if it is stable, its inverse exists and it is also stable.

We consider the signature matrix

$$
J_{\sigma, n, m}=\left[\begin{array}{cc}
I_{n} & 0 \\
0 & -\sigma^{2} I_{m}
\end{array}\right],
$$

where $n, m \in \mathbb{N}$ and $\sigma$ a strictly positive real number. Sometimes we simply use $J$ without indices.

Definition 2.1 Let $Z=Z^{\sim} \in \hat{\mathcal{W}}$. $Z$ has a $J$-spectral factorization if there exists a bistable matrix-valued function $V$ such that

$$
Z(s)=V^{\sim}(s) J V(s) \text { for all } s \in j \mathbb{R} .
$$

Such a matrix $V$ is called $J$-spectral factor of the matrix-valued function $Z$.

Definition 2.2 A vector $u$ is an equalizing vector for the matrix-valued function $Z \in \hat{\mathcal{W}}$ if $u$ is a nonzero element of $\mathrm{H}_{2}$ and $\mathrm{Zu}$ is in $\mathrm{H}_{2}^{\perp}$.

The following theorem gives equivalent conditions for the existence of a $J$-spectral factorization for a matrix-function $Z=Z^{\sim} \in \hat{\mathcal{W}}$. The proof can be found in [14].

Theorem 2.3 Let $Z=Z^{\sim} \in \hat{\mathcal{W}}$ be such that $\operatorname{det} Z(s) \neq 0$, for all $s \in j \mathbb{R} \cup\{\infty\}$. The following statements are equivalent

a. $Z$ admits a J-spectral factorization;

b. Z has no equalizing vectors;

In order to prove the main result of this paper we need the following technical lemma.

Lemma 2.4 Let $P \in \hat{\mathcal{W}}^{\left(n_{w}+n_{z}\right) \times\left(n_{y}+n_{z}\right)}$, and suppose that

$$
P^{\sim}(j \omega) J_{\sigma, n_{w}, n_{z}} P(j \omega)=J_{n_{y}, n_{z}}, \text { for almost all } \omega \in \mathbb{R} .
$$

Consider the equality

$$
\left[\begin{array}{l}
X_{1} \\
X_{2}
\end{array}\right]=\left[\begin{array}{ll}
P_{11} & P_{12} \\
P_{21} & P_{22}
\end{array}\right]\left[\begin{array}{l}
Q_{1} \\
Q_{2}
\end{array}\right]
$$

with $X_{2} \in \hat{\mathcal{A}}^{n_{z} \times n_{z}}, Q_{1} \in \hat{\mathcal{A}}^{n_{y} \times n_{z}}, Q_{2} \in \hat{\mathcal{A}}^{n_{z} \times n_{z}}, P_{21} \in \hat{\mathcal{A}}^{n_{z} \times n_{y}}, P_{22} \in \hat{\mathcal{A}}^{n_{z} \times n_{z}}$. Then the following two conditions are equivalent

a. $X_{2}$ is bistable and $\left\|X_{1} X_{2}^{-1}\right\|_{\infty}<\sigma$

b. $P_{22}$ and $Q_{2}$ are bistable and $\left\|Q_{1} Q_{2}^{-1}\right\|_{\infty}<1$

For a proof of this lemma, see [15]. 


\section{The suboptimal Nehari extension problem}

The Hankel operator with symbol $G \in L_{\infty}$, is defined as

$$
H_{G}: H_{2} \rightarrow H_{2}^{\perp}, H_{G} u=\Pi_{-} G u
$$

for $u \in H_{2}$. Its adjoint is

$$
H_{G}^{*}: H_{2}^{\perp} \rightarrow H_{2}, \quad H_{G}^{*} v=\Pi_{+} G^{\sim} v,
$$

for $v \in H_{2}^{\perp}$. Here $\Pi_{+}$and $\Pi_{-}$are the orthogonal projection from $L_{2}$ to $H_{2}$ and $H_{2}^{\perp}$, respectively (see [10]).

Using the fact that the suboptimal Nehari extension problem is trivial for stable matrixvalued functions, we can restrict this problem, without loss of generality, to antistable matrixvalued functions. The following theorem is our main result.

Theorem 3.1 Let $G$ be a matrix-valued function such that $G^{\sim} \in \hat{\mathcal{A}}^{k \times m}$, and $\sigma$ a positive real number. The following statements are equivalent:

a. $\left\|H_{G}\right\|<\sigma$.

b. There exists $K(s) \in \hat{\mathcal{A}}^{k \times m}$ such that

$$
\|G+K\|_{\infty}<\sigma
$$

c. There exists $\Lambda(s) \in \hat{\mathcal{A}}^{(k \times m) \times(k \times m)}$ a J-spectral factor for

$$
W(s)=\left[\begin{array}{cc}
I_{k} & 0 \\
G^{\sim}(s) & I_{m}
\end{array}\right]\left[\begin{array}{cc}
I_{k} & 0 \\
0 & -\sigma^{2} I_{m}
\end{array}\right]\left[\begin{array}{cc}
I_{k} & G(s) \\
0 & I_{m}
\end{array}\right]
$$

with $\Lambda_{11}^{-1}(s) \in \hat{\mathcal{A}}^{k \times k}$

Furthermore, all solutions for the suboptimal Nehari extension problem are parametrized by

$$
K(s)=X_{1}(s) X_{2}(s)^{-1},
$$

where

$$
\left[\begin{array}{l}
X_{1}(s) \\
X_{2}(s)
\end{array}\right]=\Lambda(s)^{-1}\left[\begin{array}{c}
Q(s) \\
I_{m}
\end{array}\right]
$$

with $Q(s) \in \hat{\mathcal{A}}^{k \times m},\|Q\|_{\infty}<1$.

Remark 3.2 The equivalence between the first two items is well-known. We only present the proof of the equivalence between the items $b$. and $c$.

Proof: $b . \Rightarrow c$. It is easy to see that $W(s)=W^{\sim}(s)$, and $\operatorname{det} W(s) \neq 0$ for all $s \in j \mathbb{R} \cup\{\infty\}$. In order to prove that the matrix-valued function $W(s)$ has a $J$-spectral factorization it is sufficient to show that $W(s)$ has no equalizing vectors (see Theorem 2.3).

Let $u$ be an equalizing vector for the matrix-valued function $W(s)$. This means that

$$
u=\left[\begin{array}{l}
u_{1} \\
u_{2}
\end{array}\right] \in H_{2}, u \neq 0, W u=\left[\begin{array}{l}
v_{1} \\
v_{2}
\end{array}\right] \in H_{2}^{\perp} .
$$


So, we have that

$$
\begin{aligned}
{\left[\begin{array}{l}
v_{1} \\
v_{2}
\end{array}\right] } & =W u=\left[\begin{array}{cc}
I_{k} & 0 \\
G^{\sim} & I_{m}
\end{array}\right]\left[\begin{array}{cc}
I_{k} & 0 \\
0 & -\sigma^{2} I_{m}
\end{array}\right]\left[\begin{array}{cc}
I_{k} & G \\
0 & I_{m}
\end{array}\right]\left[\begin{array}{l}
u_{1} \\
u_{2}
\end{array}\right] \\
& =\left[\begin{array}{cc}
I_{k} & G \\
G^{\sim} & G^{\sim} G-\sigma^{2} I_{m}
\end{array}\right]\left[\begin{array}{l}
u_{1} \\
u_{2}
\end{array}\right],
\end{aligned}
$$

which is equivalent to

$$
\left\{\begin{array}{c}
u_{1}+G u_{2}=v_{1}, \\
G^{\sim} u_{1}+G^{\sim} G u_{2}-\sigma^{2} u_{2}=v_{2} .
\end{array}\right.
$$

In the first equality we split $G u_{2}$ using the projections $\Pi_{-}$and $\Pi_{+}$. We obtain that

$$
\left\{\begin{array}{c}
u_{1}+\Pi_{+} G u_{2}=v_{1}-\Pi_{-} G u_{2} \\
G^{\sim}\left(u_{1}+G u_{2}\right)-\sigma^{2} u_{2}=v_{2} .
\end{array}\right.
$$

From (6) and the definition of the projection operators we have that the left-hand side of the first equality lies in $H_{2}$ and the right-hand side lies in $H_{2}^{\perp}$. This implies that

$$
u_{1}+\Pi_{+} G u_{2}=0 \text { and } v_{1}-\Pi_{-} G u_{2}=0 .
$$

Now we can replace $u_{1}$ in the second equality of $(7)$ by $-\Pi_{+} G u_{2}$. Splitting the term $G^{\sim} \Pi_{-} G u_{2}$ according to the projections, we obtain that

$$
\begin{aligned}
G^{\sim} \Pi_{-} G u_{2}-\sigma^{2} u_{2}=v_{2} & \Leftrightarrow \quad \Pi_{-} G^{\sim} \Pi_{-} G u_{2}+\Pi_{+} G^{\sim} \Pi_{-} G u_{2}-\sigma^{2} u_{2}=v_{2} \\
& \Leftrightarrow \quad \Pi_{+} G^{\sim} \Pi_{-} G u_{2}-\sigma^{2} u_{2}=v_{2}-\Pi_{-} G^{\sim} \Pi_{-} G u_{2} .
\end{aligned}
$$

Using similar arguments as before, we have that

$$
v_{2}=\Pi_{-} G^{\sim} \Pi_{-} G u_{2}
$$

and

$$
\Pi_{+} G^{\sim} \Pi_{-} G u_{2}-\sigma^{2} u_{2}=0
$$

which is equivalent to

$$
\left(H_{G}^{*} H_{G}-\sigma^{2} I_{m}\right) u_{2}=0 .
$$

Since $b$. holds, we have that $a$. holds, and thus we obtain that $u_{2}$ must be zero. From (8) we see that also $u_{1}$ must be zero as well, so $u=0$. We conclude that the matrix-valued function $W$ has no equalizing vectors, which by Theorem 2.3 implies that $W$ has a $J$-spectral factorization.

Let $\Lambda$ be a $J$-spectral factor. We prove that $\Lambda_{11}(s)^{-1}$ is a stable matrix-valued function. The following equality holds

$$
\left[\begin{array}{c}
G+K \\
I
\end{array}\right]=\left[\begin{array}{cc}
I & G \\
0 & I
\end{array}\right]\left[\begin{array}{c}
K \\
I
\end{array}\right]=\left[\begin{array}{cc}
I & G \\
0 & I
\end{array}\right] \Lambda^{-1} \Lambda\left[\begin{array}{c}
K \\
I
\end{array}\right]=\left[\begin{array}{ll}
P_{11} & P_{12} \\
P_{21} & P_{22}
\end{array}\right]\left[\begin{array}{l}
Q_{1} \\
Q_{2}
\end{array}\right]
$$

where

$$
\begin{aligned}
{\left[\begin{array}{ll}
P_{11} & P_{12} \\
P_{21} & P_{22}
\end{array}\right] } & =\left[\begin{array}{cc}
I & G \\
0 & I
\end{array}\right] \Lambda^{-1} \text { and } \\
{\left[\begin{array}{l}
Q_{1} \\
Q_{2}
\end{array}\right] } & =\Lambda\left[\begin{array}{c}
K \\
I
\end{array}\right]
\end{aligned}
$$


with $P_{21}, P_{22}, Q_{1}$ and $Q_{2}$ stable matrix-valued functions. Now, by the definition of $\Lambda$,

$$
P^{\sim} J_{\sigma, k, m} P=\left(\Lambda^{-1}\right)^{\sim}\left[\begin{array}{cc}
I & G \\
0 & I
\end{array}\right]^{\sim} J_{\sigma, k, m}\left[\begin{array}{cc}
I & G \\
0 & I
\end{array}\right] \Lambda^{-1}=J .
$$

Combining this with (3), we conclude from Lemma 2.4 that $P_{22}$ is bistable. Using matrix block manipulation, it can be proved that $\Lambda_{11}^{-1}=V_{11}-V_{12} P_{22}^{-1} V_{21}$, where $V=\Lambda^{-1}$. Since all the elements expressing $\Lambda_{11}^{-1}$ are stable, we have that $\Lambda_{11}^{-1}$ is also stable.

Applying Lemma 2.4 once more, we obtain that $Q_{2}$ is a bistable matrix-valued function. Multiplying the relation (12) to the left with $\Lambda^{-1}$ and to the right with $Q_{2}^{-1}$ we have that

$$
\left[\begin{array}{c}
K Q_{2}^{-1} \\
Q_{2}^{-1}
\end{array}\right]=\Lambda\left[\begin{array}{c}
Q_{1} Q_{2}^{-1} \\
I
\end{array}\right]
$$

Denoting $X_{1}=K Q_{2}^{-1}$ and $X_{2}=Q_{2}^{-1}$, gives

$$
X_{1} X_{2}^{-1}=K Q_{2}^{-1} Q_{2}=K
$$

and, using (13), $X_{1}$ and $X_{2}$ satisfy (5), with $Q=Q_{1} Q_{2}^{-1}$.

$c . \Rightarrow b$. Suppose that there exists a $J$-spectral factor $\Lambda$ for the matrix-valued function $W$ such that $\Lambda_{11}$ is bistable. Let $V$ denote $\Lambda^{-1}$. Using matrix block manipulation, it can be proved that

$$
V_{22}(s)^{-1}=\Lambda_{22}(s)-\Lambda_{21}(s) \Lambda_{11}(s)^{-1} \Lambda_{12}(s) .
$$

Since $\Lambda_{22}(s), \Lambda_{21}(s), \Lambda_{11}(s)^{-1}$ and $\Lambda_{12}(s)$ are stable, also $V_{22}(s)^{-1}$ is stable. So, we conclude that $V_{22}$ is a bistable matrix-valued function. If we define $K_{0}=V_{12} V_{22}^{-1}$, then $K_{0}$ is stable. Furthermore, from the equality

$$
\left[\begin{array}{c}
G+K_{0} \\
I_{m}
\end{array}\right]=\left[\begin{array}{cc}
I_{k} & G \\
0 & I_{m}
\end{array}\right] V\left[\begin{array}{c}
0 \\
V_{22}^{-1}
\end{array}\right]
$$

and Lemma 2.4, we see that $K_{0}$ is a solution for the suboptimal Nehari extension problem.

Using again Lemma 2.4, it is easy to see that any $K=X_{1} X_{2}^{-1}$, where $X_{1}$ and $X_{2}$ are given by (5), is a solution for the suboptimal Nehari extension problem.

Remark 3.3 The results stated in this paper hold also for $\hat{\mathcal{W}}_{-}$. This is the class of transfer functions obtained similarly as $\hat{\mathcal{W}}$. The impulse responses in $\mathcal{A}_{-}$are the sum of a weighted $L_{1}$-function with a delta function. This is the same class as the stable one considered in [5].

Remark 3.4 In case that the matrix-valued function $W(s)$, defined in (4) admits a J-spectral factorization, we can construct a J-spectral factor using the procedure described in [12], [13], [14]. The disadvantage of the method used there is that it relays on the existence of solutions for two equations involving projection operators.

Corollary 3.5 Let $u=\left[\begin{array}{l}u_{1} \\ u_{2}\end{array}\right] \in H_{2}$ be an equalizing vector for the matrix-valued function $W(s)$ defined in (4). The following assertions hold:

a. $u$ has the following representation

$$
u=\left[\begin{array}{c}
-\Pi_{+} G u_{2} \\
u_{2}
\end{array}\right] .
$$


b. $u_{2}$ is an eigenvector for the compact nonnegative operator $H_{G}^{*} H_{G}$ corresponding to the eigenvalue $\sigma^{2}$. Moreover, $u_{2}$ can be choosen to have unitary norm.

c. If $v=W u \in H_{2}^{\perp}$, then

$$
v=\left[\begin{array}{l}
v_{1} \\
v_{2}
\end{array}\right]=\left[\begin{array}{c}
H_{G} u_{2} \\
\Pi_{-} G^{\sim} H_{G} u_{2}
\end{array}\right]
$$

d. $\left(u_{2}, \frac{v_{1}}{\sigma}\right)$ is a Schmidt pair corresponding to $\sigma$, a nonzero singular value of the Hankel operator with symbol $G$.

Proof: $a$. Using (8), we see that $u$ has the representation (14).

$b$. Let $u=\left[\begin{array}{l}u_{1} \\ u_{2}\end{array}\right] \in H_{2}$ be an equalizing vector for the matrix-valued function $W(s)$, defined in (4). From (10), we see that $u_{2}$ is an eigenvector for $H_{G}^{*} H_{G}$ corresponding to the eigenvalue $\sigma^{2}$, and that, without lost of generality, $u_{2}$ can be choosen to have unitary norm.

c. From (8), (9), and the definition of the Hankel operator, we obtain the representation (15) for the vector $v=W u$.

d. From (15) we see that $v_{1}=H_{G} u_{2}$, so

$$
H_{G} u_{2}=\sigma \frac{v_{1}}{\sigma}
$$

and using b.

$$
H_{G}^{*}\left(\frac{v_{1}}{\sigma}\right)=H_{G}^{*}\left(\frac{H_{G} u_{2}}{\sigma}\right)=\frac{1}{\sigma} H_{G}^{*} H_{G} u_{2}=\sigma u_{2} .
$$

Corollary 3.6 If $(\phi, \psi)$ is the Schmidt pair of the Hankel operator with symbol G corresponding to a nonzero singular value $\sigma$, then

$$
u=\left[\begin{array}{c}
-\Pi_{+} G \phi \\
\phi
\end{array}\right]
$$

is an equalizing vector for the matrix-valued function $W(s)$ defined in (4), and

$$
W u=\sigma\left[\begin{array}{c}
\psi \\
\Pi_{-} G^{\sim} \psi
\end{array}\right] .
$$

Proof: Let $(\phi, \psi)$ be the Schmidt pair of the Hankel operator with symbol $G$ corresponding to a nonzero singular value $\sigma$. We have the following sequence of equalities:

$$
\begin{aligned}
W u & =\left[\begin{array}{cc}
I_{k} & G \\
G^{\sim} & G^{\sim} G-\sigma^{2} I_{m}
\end{array}\right]\left[\begin{array}{c}
-\Pi_{+} G \phi \\
\phi
\end{array}\right] \\
& =\left[\begin{array}{c}
-\Pi_{+} G \phi+G \phi \\
-G^{\sim} \Pi_{+} G \phi+G^{\sim} G \phi-\sigma^{2} \phi
\end{array}\right] \\
& =\left[\begin{array}{c}
\Pi_{-} G \phi \\
G^{\sim} \Pi_{-} G \phi-\sigma^{2} \phi
\end{array}\right] \\
& =\left[\begin{array}{c}
\Pi_{-} G \phi \\
\Pi_{-} G^{\sim} \Pi_{-} G \phi+\left(\Pi_{+} G^{\sim} \Pi_{-} G \phi-\sigma^{2} \phi\right)
\end{array}\right]
\end{aligned}
$$




$$
\begin{aligned}
& =\left[\begin{array}{c}
\Pi_{-} G \phi \\
\Pi_{-} G^{\sim} \Pi_{-} G \phi+\left(H_{G}^{*} H_{G} \phi-\sigma^{2} \phi\right)
\end{array}\right] \\
& =\left[\begin{array}{c}
\Pi_{-} G \phi \\
\Pi_{-} G^{\sim} \Pi_{-} G \phi
\end{array}\right] \\
& =\left[\begin{array}{c}
H_{G} \phi \\
\Pi_{-} G^{\sim} H_{G} \phi
\end{array}\right] \in H_{2}^{\perp} .
\end{aligned}
$$

This shows that $u$ is an equalizing vector for the matrix-valued function $W(s)$ defined in (4). Since $(\phi, \psi)$ is the Schmidt pair of the Hankel operator with symbol $G$ corresponding to the nonzero singular value $\sigma$, we have that $H_{G} \phi=\sigma \psi$. So, the relation (16) is satisfied.

\section{The Nehari extension problem and equalizing vectors}

The problem of finding $K \in H_{\infty}^{k \times m}$ that achieve the minimum distance in

$$
\inf _{K \in H_{\infty}^{k \times m}}\|G+K\|_{\infty}=\left\|H_{G}\right\|
$$

is called the Nehari extension problem. The following theorems give connections between the equalizing vectors and the solutions of the Nehari extension problem.

Theorem 4.1 Suppose that $G \in \hat{\mathcal{W}}^{k \times m}$. Then any $K_{0} \in H_{\infty}^{k \times m}$ solving the Nehari extension problem, that is, satisfying

$$
\left\|G+K_{0}\right\|_{\infty}=\left\|H_{G}\right\|
$$

also satisfies

$$
\left(G+K_{0}\right) u_{2}=H_{G} u_{2},
$$

where $u_{2}$ is an eigenvector for the compact nonnegative operator $H_{G}^{*} H_{G}$ corresponding to the largest eigenvalue $\left\|H_{G}\right\|^{2}$. Moreover, $G+K_{0}$ has constant modulus almost everywhere on the imaginary axis.

Proof: We have that the Hankel operator with symbol $G$ is a compact operator (see [8], Lemma 8.1.7), and the equality

$$
\left\|H_{G} u_{2}\right\|_{H_{2}^{\perp}}=\left\|H_{G}\right\|\left\|u_{2}\right\|_{H_{2}}
$$

holds (see [8], Lemma 8.1.12). The rest of the proof follows from Theorem 8.1.11 in [8].

The following theorem provides a connection between the equalizing vectors and solutions of the Nehari extension problem.

Theorem 4.2 Let $\sigma=\left\|H_{G}\right\|$. Suppose that $G \in \hat{\mathcal{W}}^{k \times m}$ is a given matrix-valued function and that $u=\left[\begin{array}{l}u_{1} \\ u_{2}\end{array}\right] \in H_{2}$ is an equalizing vector for the matrix-valued function $W(s)$, defined in (4). If there exists a solution $K_{0}$ of the Nehari extension problem, then on the imaginary axis it satisfies

$$
K_{0} u_{2}=u_{1}
$$


Proof: Let $u=\left[\begin{array}{l}u_{1} \\ u_{2}\end{array}\right] \in H_{2}$ be an equalizing vector for the matrix-valued function $W(s)$, defined in (4). By Corollary 3.5b we know that $u_{2}$ is an eigenvector corresponding to the eigenvalue $\left\|H_{G}\right\|^{2}$. If $K_{0}$ is a solution for the Nehari extension problem, then by Theorem 4.1 it must satisfy

$$
\left(G+K_{0}\right) u_{2}=H_{G} u_{2}
$$

which is equivalent to

$$
\begin{array}{rlr}
K_{0} u_{2} & =-G u_{2}+\Pi_{-} G u_{2} \\
& =-\Pi_{+} G u_{2} \\
& =u_{1} \quad \text { from (14) }
\end{array}
$$

So, the equality (20) holds.

Remark 4.3 From the relation (20) one can see that the equalizing vector is fixing the solution of the Nehari extension problem in the direction of the eigenvector corresponding to the largest singular value of the Hankel operator with symbol $G$.

If the symbol is a scalar function, an equalizing vector can be used to prove the uniqueness of the solution for the Nehari extension problem.

Corollary 4.4 Consider the scalar transfer function $g \in \hat{\mathcal{W}}$ and let $\sigma=\left\|H_{G}\right\|$. Suppose that $u=\left[\begin{array}{l}u_{1} \\ u_{2}\end{array}\right] \in H_{2}$ is an equalizing vector for the matrix-valued function $W(s)$, defined in (4). If there exists a solution $k_{0}$ of the Nehari extension problem, it is unique, and on the imaginary axis it is given by

$$
k_{0}=\frac{u_{1}}{u_{2}}
$$

Proof: Since $u_{2} \in H_{2}$, it is zero, at most, on a set of measure zero (see [8], Lemma A.6.20) of the imaginary axis. This means that we can divide the equality (20) through $u_{2}$ and obtain (21).

Remark 4.5 For the scalar case, the previous corollary gives the solution for the Nehari extension problem, providing that we have an equalizing vector. From Theorem 4.1 we have that $g+k_{0}$ has constant modulus almost everywhere on the imaginary axis. This means that once we have an equalizing vector, we find a $k_{0}$ which "equalizes" $g$ over the imaginary axis (complete $g$ to a function of constant modulus almost everywere on the imaginary axis).

Remark 4.6 The Nehari extension problem corresponding to every $G \in \hat{\mathcal{A}}^{k \times m}$ has a unique solution. Let us denote it by $K$. If $\left(\sigma_{n}\right)_{n \in \mathcal{N}}$ is a decreasing sequence with limit $\left\|H_{G}\right\|$ and $\left(K_{n}\right)_{n \in \mathcal{N}}$ is a corresponding sequence of solutions (can be choosen rational) for the suboptimal Nehari extension problems, then there exists a subsequence $K_{\alpha(n)}$ such that

$$
\lim _{n \rightarrow \infty}\left\langle K_{\alpha(n)} f(s), g(s)\right\rangle_{L_{2}}=\langle K f(s), g(s)\rangle_{L_{2}}
$$

for every $f \in L_{2}^{m}$ and every $g \in L_{2}^{k}$.

A proof for the results stated in the previous remark can be found in [8], Theorem 8.3.8. 


\section{References}

[1] V.M. Adamjan, D.Z. Arov, and M.G. Krein. Infinite Hankel block matrices and related extension problems. American Mathematical Society Translations, 111:133-156, 1978.

[2] J.A. Ball and J.W. Helton. A Beurling-Lax theorem for the Lie group $U(m, n)$ which contains most classical interpolation theory. Journal of Operator Theory, 9:107-142, 1983.

[3] J.A. Ball and J.W. Helton. Beurling-Lax representation using classical Lie groups with many applications iii: Groups preserving two bilinear forms. Amer. Journal Math., 108:95-174, 1986.

[4] F.M. Callier and J. Winkin. On spectral factorization and lq-optimal regulation for multivariable distributed systems. Technical report, Faculty of Mathematical Sciences, 1989. Belgium, Namur.

[5] R.F. Curtain and M. Green. Analytic systems problems and J-lossless coprime factorizations for infinite-dimensional linear systems. Linear Algebra and its Applications, 257:121-161, 1997.

[6] R.F. Curtain and A. Ran. Explicit formulas for Hankel norm approximations of infinitedimensional systems. Integral Equations and Operator Theory, 13:455-469, 1989.

[7] R.F. Curtain and H.J. Zwart. The Nehari problem for the Pritchard-Salamon class of infinite-dimensional linear systems: a direct approach. Integral Equations and Operator Theory, 18:130-153, 1994.

[8] R.F. Curtain and H.J. Zwart. An Introduction to Infinite-Dimensional Linear Systems Theory. Springer-Verlag, New York, 1995.

[9] C. Foias and A. Tannenbaum. On the Nehari problem for a certain class of $L_{\infty}$-functions appearing in control theory. Journal of Functional Analysis, 74:146-159, 1987.

[10] B. Francis. A Course in $H_{\infty}$ Control Theory, volume 88 of Lecture Notes in Control and Information Science. Springer-Verlag, Berlin, 1987.

[11] M. Green, K. Glover, D. Limebeer, and J. Doyle. A J-spectral factorization approach to $H_{\infty}$ control. SIAM Journal on Control and Optimization, 28:1350-1371, 1990.

[12] O.V. Iftime and H.J. Zwart. $J$-spectral factorization and equalizing vectors. Technical report, 1549, University of Twente, September 2000.

[13] O.V. Iftime and H.J. Zwart. The standard $H_{\infty}$-suboptimal control problem for $L T I$ infinite-dimensional systems. Technical report, 1532, University of Twente, July 2000.

[14] O.V. Iftime and H.J. Zwart. J-spectral factorization and equalizing vectors. Systems and Control Letters, Accepted for publication.

[15] G. Meinsma and H. Zwart. On $H_{\infty}$ control for dead-time systems. IEEE Transactions on Automatic Control, 45:272-285, 2000. 
[16] A. Ran. Hankel norm approximation for infinite-dimensional systems and Wiener-Hopf factorization. In R.F. Curtain, editor, Modelling Robustness and Sensitivity Reduction in Control Systems, NATO ASI Series, pages 57-70. Springer-Verlag, 1986.

[17] A.J. Sasane and R.F. Curtain. Optimal Hankel norm approximation for infinitedimensional systems. In Proceedings of the MTNS 2000, Perpignan, France, 2000. to appear.

[18] A.J. Sasane and R.F. Curtain. Sub-optimal Hankel norm approximation for the analytic class of infinite-dimensional systems (extended version). Technical report, IWI 2000-4-01, University of Groningen, 2000. Available at http://www/math.rug.nl/ amols/index.html.

[19] A.J. Sasane and R.F. Curtain. Optimal Hankel norm approximation for the PritchardSalamon class of infinite-dimensional systems. Integral Equations and Operator Theory, To appear. 